\title{
Diabetes insipidus and hypopituitarism in HIV: an unexpected cause
}

\author{
Carlos Tavares Bello, Francisco Sousa Santos, João Sequeira Duarte and \\ Carlos Vasconcelos
}

Endocrinology Department, Hospital de Egas Moniz, Lisboa, Portugal

\author{
Correspondence \\ should be addressed \\ to C Tavares Bello \\ Email \\ bello_carlos4@yahoo.com
}

\section{Summary}

Central diabetes insipidus (DI) is a rare clinical entity characterized by low circulating levels of antidiuretic hormone $(A D H)$ presenting with polyuria and volume depletion. Pituitary surgery is the most common cause of central DI in adults. Pituitary and hypothalamic disease, particularly invasive neoplasms, rarely cause DI, being idiopathic cases responsible for the majority of non-surgical cases. HIV patients, especially those with poor virulogical control, are prone to the development of CNS neoplasms, particularly lymphomas. These neoplasms usually become manifest with mass effects and seizures. Central DI and hypopituitarism are uncommon initial manifestations of primary CNS lymphomas. The authors describe the case of 29-year-old female, HIV-positive patient whose CNS lymphoma presented with DI.

\section{Learning points:}

- Central diabetes insipidus has multiple causes and central nervous system lymphomas are not often considered in the differential diagnosis due to their low prevalence.

- Accurate biochemical diagnosis should always be followed by etiological investigation.

- The HIV population is at risk for many neoplasms, especially CNS lymphomas.

- New-onset polyuria in an HIV-positive patient in the absence of focal neurological signs should raise the suspicion for a central nervous system process of neoplastic nature.

- This clinical entity usually constitutes a therapeutical challenge, often requiring a multidisciplinary approach for optimal outcome.

\section{Background}

Central diabetes insipidus (DI) is a rare hypothalamicpituitary disease with an estimated prevalence of $3 / 100000$ (1). It results from an inherited or acquired antidiuretic hormone deficiency usually secondary to supraoptic and/or paraventricular hypothalamic nuclei dysfunction. In $30 \%$ of the cases, no identifiable cause is found (idiopathic DI), while space-occupying lesions (inflammatory and neoplastic), cranial surgery and traumatic brain injury, account for the majority of the non-idiopathic cases (2). Primary central nervous system lymphoma (PCNSL) is a somewhat frequent neoplasm in the HIV population, often presenting with altered mental status, focal neurological signs or encephalopathy due to its frequent multifocal nature (3). The prognosis is usually poor, even with timely introduction of highly active antiretroviral therapy (HAART), chemo and radiation therapy. Diabetes insipidus is not considered a typical presenting feature of PCNSL. Previous case reports often refer the occurrence of polyuria or 'urinary incontinence' later in the disease course, being frequently preceded by confusion, epileptic seizures and/or focal neurological signs (4). An accurate biochemical diagnosis, followed by etiological investigation is required in order to decide the best treatment options. A multidisciplinary approach 
is needed due to the high disease complexity and poor prognosis. The authors report a case of a 29-year-old female patient presenting with diabetes insipidus as the initial manifestation of a primary CNS lymphoma.

\section{Case presentation}

We report a case of a 29-year-old female, with a known medical history of active smoking (12 pack/year), no alcohol or substance abuse and HIV infection (diagnosed at the age of 21 years). The patient had irregular medical follow-up and did not take regular medication, namely antiretroviral therapy. The patient had undergone two full-term pregnancies with no complications or excessive post-partum bleeding. She recalled an inability to breastfeed after the second birth, despite preservation of regular menses. No headaches, visual field defects or any other clinical or laboratory suggestions of hypopituitarism were present.

Before hospital admission, the patient described polyuria (allegedly more than $4 \mathrm{~L} /$ day), polydipsia (normal thirst perception) and malaise for one month subsequently followed by nausea and vomiting. No visual symptoms were present.

At the Emergency Department, the patient presented with severe clinical dehydration, hypotension (BP $88 / 63 \mathrm{mmHg}$ ), elevated heart rate (100 beats per minute), tympanic temperature of $35.8^{\circ} \mathrm{C}$, altered mental status (Glasgow Coma Scale 14 - confused) along with bilateral axillary non-tender elastic lymphadenopathy.

\section{Investigation}

Initial laboratory investigation is presented in Table 1.

After admission, in the medical ward, polyuria was confirmed by a daily urine output of $6000 \mathrm{~mL}$, corresponding to a negative water balance of $3000 \mathrm{~mL}$ $(66 \mathrm{~mL} / \mathrm{kg} /$ day $)$. The confirmed polyuria, elevated serum osmolality along with inadequately low urinary osmolality and normoglycemia were diagnostic for diabetes insipidus. A water deprivation test was not performed due to the clinical severity and spontaneous diagnostic laboratory abnormalities. After establishing the diagnosis of DI, the distinction between a central from a nephrogenic mechanism was achieved by a desmopressin challenge test: after administration of 5 units of desmopressin subcutaneously, diuresis progressively decreased over a 4 -h period and the subsequent 24-h water balance became almost neutral $(-200 \mathrm{~mL})$. Serum osmolality normalized, and
Table 1 Laboratory investigations at admission.

\begin{tabular}{l}
\hline Laboratory parameters \\
\hline Hemoglobin $(\mathrm{g} / \mathrm{dL})$ \\
Leucocytes $\left(10^{6} / \mathrm{L}\right)$ \\
Neutrophils $\left(10^{6} / \mathrm{L}\right)$ \\
Lymphocytes $\left(10^{6} / \mathrm{L}\right)$ \\
Eosinophils $\left(10^{6} / \mathrm{L}\right)$ \\
Platelets $\left(10^{3} / \mu \mathrm{L}\right)$ \\
Sodium $(\mathrm{mEq} / \mathrm{L})$ \\
Potassium $(\mathrm{mEq} / \mathrm{L})$ \\
Serum osmolality (mosmol/kg) \\
Urine osmolality (mosmol/kg) \\
Serum creatinine $(\mathrm{mg} / \mathrm{dL})$ \\
Serum urea $(\mathrm{mg} / \mathrm{dL})$ \\
eGFR (CKD-EPI) $\left(\mathrm{mL} / \mathrm{min} / 1.73 \mathrm{~m}^{2}\right)$ \\
Urinary density \\
LDH \\
Plasma glucose $(\mathrm{mg} / \mathrm{dL})$
\end{tabular}

\begin{tabular}{c}
\hline Value \\
\hline 11 \\
8000 \\
4300 \\
1600 \\
50 \\
100 \\
154 \\
3.8 \\
340 \\
78 \\
1.98 \\
90 \\
34 \\
1003 \\
803 \\
65 \\
\hline
\end{tabular}

\begin{tabular}{c}
\hline Normal range \\
\hline $12-14$ \\
$4000-10000$ \\
$2500-7500$ \\
$1500-3500$ \\
$40-400$ \\
$150-400$ \\
$135-145$ \\
$3.5-5.2$ \\
$275-295$ \\
$>300$ \\
$<1.2$ \\
$<42$ \\
$>90$ \\
$1010-1020$ \\
$140-280$ \\
$60-90$ \\
\hline
\end{tabular}

urinary osmolality rose to values above $400 \mathrm{mosmol} /$ kg. Pituitary hormonal profile was compatible with panhypopituitarism (Table 2). Regarding HIV status documented, CD4 count was $607 / \mathrm{mm}^{3}$ and a viral load of 74793 copies $/ \mathrm{mL}$.

The patient underwent central nervous system cross-sectional imaging, initially with CT followed by a magnetic resonance to better characterize the lesion. A space occupying lesion $(30 \times 25 \mathrm{~mm})$ with regular borders expanding to the supraselar and interpeduncular spaces compressing the hypothalamus was evident along with the absent bright spot of the neurohypophysis (Figs. 1 and 2).

Cerebrospinal fluid (CSF) analysis (flow cytometry) documented large atypical B cells (28.5\%) positive for CD20, CD10, CD38 and CD81. Epstein-Barr virus polymerase chain reaction (PCR) analysis of the CSF was negative. Excisional axillary lymph node biopsy further showed malignant lymphoid cell infiltration. Peripheral blood cell immunophenotyping, bone marrow biopsy

Table 2 Baseline pituitary profile.

\begin{tabular}{|c|c|c|}
\hline Laboratory parameters & Value & Reference range \\
\hline Morning plasma cortisol ( $\mu \mathrm{g} / \mathrm{dL})$ & 3.63 & $10-20$ \\
\hline ACTH $(p g / m L)$ & $<5$ & $<46$ \\
\hline TSH $(\mu \mathrm{U} / \mathrm{L})$ & 0.22 & $0.5-4.68$ \\
\hline Free T4 (pmol/L) & 8 & $12-22.8$ \\
\hline Prolactin (ng/mL) & 41.9 & $<21$ \\
\hline $\mathrm{FSH}(\mathrm{U} / \mathrm{L})$ & $<0.66$ & $1.5-12.4$ \\
\hline $\mathrm{LH}(\mathrm{U} / \mathrm{L})$ & $<0.22$ & $1.7-8.6$ \\
\hline Estradiol (pg/mL) & 10.9 & $27-123$ \\
\hline IGF-1 (ng/mL) & $<25$ & $91-282$ \\
\hline
\end{tabular}




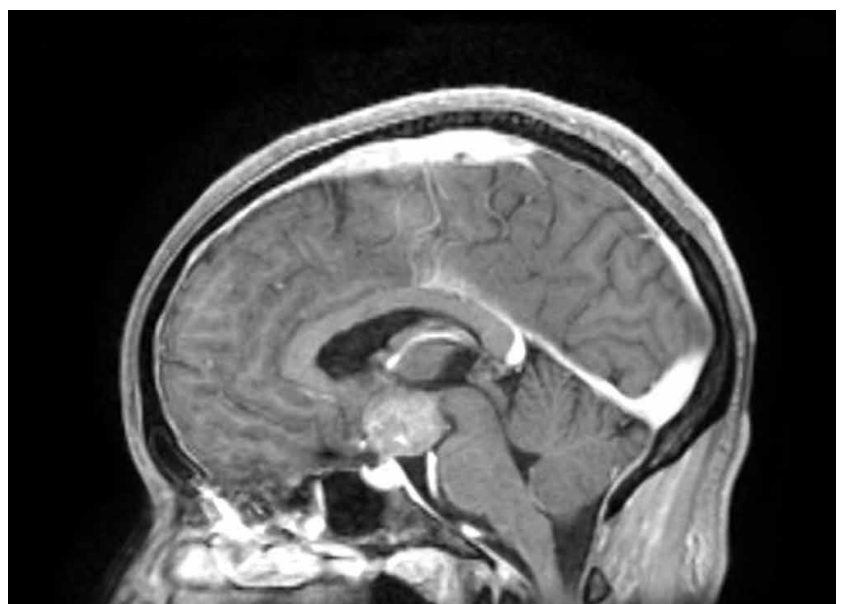

Figure 1

CNS T1-weighted contrast-enhanced MRI showing a smooth shaped suprasellar compressing the hypophysis and hypothalamus and with an absent neurohypophyseal bright spot.

and cross-sectional imaging of chest, abdomen and pelvis were unremarkable.

The finding of a single large CNS mass along with isolated axillary lymph node involvement and no other evidence for disease led to the diagnosis of advanced (stage IV) primary CNS lymphoma (diffuse large B cell lymphoma subtype) with hypopituitarism and diabetes insipidus.

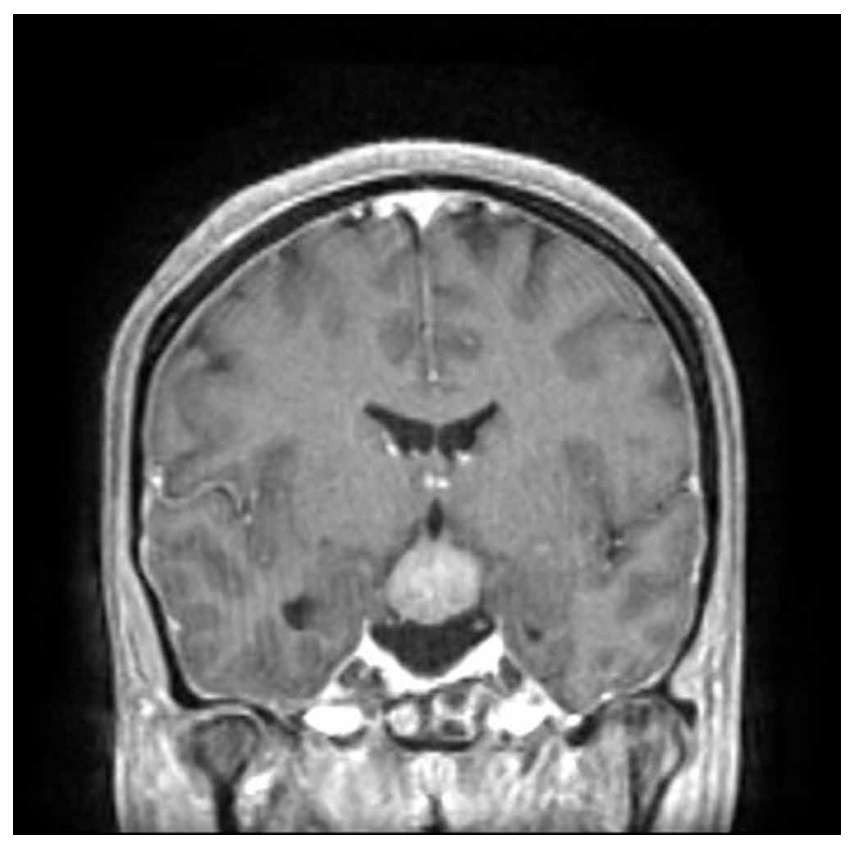

Figure 2

CNS T1-weighted contrast-enhanced MRI showing interpenduncular spaces compressing the hypophysis and hypothalamus and with an absent neurohypophyseal bright spot.

\section{Treatment}

Patient was soon started on hormone replacement therapy with hydrocortisone thrice daily $(25 \mathrm{mg} /$ day), levothyroxine $(75 \mu \mathrm{g} /$ day $)$ and intranasal desmopressin (twice daily and as needed), which significantly improved the patient's clinical status. Regarding HIV, highly active antiretroviral therapy with abacavir, lamivudine and raltegravir were also timely initiated. Chemotherapy was prescribed as soon as the lymphoma diagnosis was established and the regimen included intrathecal methotrexate and CHOP-R (cyclophosphamide, doxorubicin, vincristine, prednisolone and rituximab). She completed one cycle of R-CHOP and 9 intrathecal methotrexate treatments.

\section{Outcome and follow-up}

Despite adequate hormonal treatment, significant ionic and urine volume oscillations were documented. Chemotherapy along with concurrent hypokalemia and abacavir rendered the patient unresponsive to desmopressin (combined central and nephrogenic DI) creating a significant clinical and analytical instability. Although hypokalemia correction led to partial polyuria improvement, chemotherapy-related toxicity resulted in grade 4 mucositis that progressed to septic shock and disseminated intravascular coagulation. The latter complication resulted in the patient's demise 2 months after the lymphoma diagnosis.

\section{Discussion}

Diabetes insipidus (DI) is a rare clinical entity characterized by hypo-osmolar polyuria, due to an insufficient secretion of $\mathrm{ADH}$ (antidiuretic hormone - arginine vasopressin) (i.e. central diabetes insipidus) or renal insensitivity to $\mathrm{ADH}$ action in the kidneys (i.e. nephrogenic diabetes insipidus). $\mathrm{ADH}$ is synthetized in the supraoptic and paraventricular nuclei of the hypothalamus and is carried along with neurophysin down the pituitary stalk to be released into the pituitary portal circulation at the level of the neurohypophysis. Its production is regulated by minimal changes of plasma osmolality that are sensed by the osmoreceptors in the organum vasculosum of the lamina terminalis that lies outside the blood-brain barrier. Intravascular volume status, blood pressure and glucocorticoids also influence ADH secretion, although to a lesser degree than serum osmolality. ADH acts on the renal collecting tubule V2 receptors, and it reduces free 
water clearance, therefore preventing dehydration. Ionic balance, namely sodium levels, is highly regulated by this system. Disease processes that disrupt ADH homeostasis will have significant biochemical repercussions with potentially serious clinical consequences.

Central DI may be caused by CNS neoplasms (25\%), neurosurgery (20\%) and head trauma (16\%) and granulomatous diseases $(<5 \%)$ (5). In up to $30 \%$ of the cases, the etiology remains unknown (idioipathic); however, with the relatively recent discovery of anti$\mathrm{ADH}$-secreting cell antibodies, around one third of the previously presumed idiopathic cases are nowadays labeled as autoimmune (6). Among neoplasms, in the reported case, the differential diagnosis included an invasive pituitary adenoma, craniopharyngeoma, glioma, meningioma and a central nervous system lymphoma. The clinical presentation made pituitary adenoma unlikely, as DI is an exceedingly rare manifestation of the latter. The imaging phenotype was not suggestive of a craniopharyngeoma or meningeoma. There is significant overlap regarding MRI findings between CNS lymphomas and gliomas; however, the age group, HIV status and CSF findings made lymphoma diagnosis emerge as a probable etiology (7).

As expected, polyuria (daily urine output of more than $40 \mathrm{~mL} / \mathrm{kg}$ body weight) and polydipsia of sudden onset are the cardinal clinical features of central DI. If compensatory water intake cannot be maintained, either due to loss of thirst perception (adipsic DI) or no access to water, severe dehydration and altered mental status will develop. Laboratory findings are usually remarkable for high serum osmolality ( $>295 \mathrm{mosmol} / \mathrm{kg}$ ) along with a low or inappropriate normal urinary osmolality $(<100 \mathrm{mosmol} / \mathrm{kg})$. Unlike the reported case, which already presented with obvious laboratory abnormalities, DI diagnosis is usually established after a medically supervised water deprivation test. The latter involves serial measurement of patient's weight, urine volume and osmolality along with plasma sodium and water after cessation of fluid intake in a controlled setting. A rise in serum osmolality without an accompanying rise of urine osmolality is diagnostic of DI. To further differentiate central from nephrogenic DI, a desmopressin challenge test is recommended. Desmopressin is a synthetic formulation of antidiuretic hormone and physiologically favors renal urinary concentrating properties. In central DI, desmopressin is expected to induce an increment in urine osmolality (more than 50\% of baseline) that supports an absolute ADH deficiency and adequate renal response to the peptide. On the other hand, the lack of urine osmolality response (U-osmol rise of less than 10\%) favors renal unresponsiveness to $\mathrm{ADH}$ and nephrogenic DI (8). Treatment of central diabetes insipidus is complex, frequently requires a fine balance between water intake, output and desmopressin dosing and should be done in a specialized setting. Outpatient desmopressin treatment includes oral and intranasal formulations. In a patient with preserved thirst mechanism and capable of maintaining an adequate water intake, as needed desmopressin administration is acceptable and it improves improved symptom control and quality of life.

HIV is an infectious disease with a relatively high prevalence in Portugal. In the past, it was considered a rapidly progressive disease with high mortality secondary to opportunistic infections. With the advent of highly active antiretroviral therapy (HAART), HIV is now a chronic disease, accounting cardiovascular disease for the majority of deaths in this population (9). First-line treatment regimens usually include nucleoside reverse transcriptase inhibitors such as Tenofovir or abacavir. This pharmacological class has been associated with both proximal renal tubular acidosis and nephrogenic diabetes insipidus. Since Tenofovir was more frequently been associated with the latter than abacavir, the authors considered the latter agent to be indicated in the reported case (10). Regarding neoplastic disease, the incidence of AIDS-defining diseases such as lymphomas (primary CNS, Burkitt and Immunoblastic subtypes), Kaposi sarcoma and invasive cervical cancers, is declining (11). Despite the availability of effective therapy in industrialized countries, treatment noncompliance may favor the occurrence of the AIDS defining neoplasms.

Primary CNS lymphomas are extra-nodal high-grade lymphoid neoplasms affecting up to $5 \%$ of patients with AIDS. Its incidence has been declining since the introduction of HAART. They usually are of the diffuse large B cell subtype (90\%). Risk factors include low CD4 counts (usually less than 30 cells $/ \mu \mathrm{L}$ ), intravenous drug use/ abuse and AIDS. It affects mostly males in the 4th decade of life. Multifocal disease is present at diagnosis in more than $50 \%$ of HIV positive patients. The multifocal nature of the disease gives rise to a distinct clinical picture, with a clinical presentation ranging from focal neurological deficits to progressive dementia, encephalopathy, stupor and even intravascular malignant lymphomatosis. CSF analysis and CNS imaging may be helpful in diagnosis; however, stereotactic brain biopsy is required for definite diagnosis. Treatment options include HAART, systemic and intrathecal chemotherapy and radiation therapy. Even with intensive treatment, prognosis remains dismal 
with an average overall survival of 18-26 months (12). CNS lymphomas in immunocompetent patients have no known risk factors. These often present at a more advanced age (6th decade), are often solitary (giving rise to focal neurological deficits) and are less aggressive, despite an average survival of up to 48 months.

Only a few case reports of CNS lymphomas presenting with DI and hypopituitarism have been described so far affecting only immunocompetent patients. Among the HIV-positive population, case reports of central DI have been attributed to criptococcal and syphilitic meningitis, and pituitary involvement by a paranasal diffuse large B cell lymphoma. The latter had a similar clinical presentation as the reported case regarding hypopituitarism and DI; however, our patient was younger (age, 29 vs 53 years) and had a worse virulogic control. In immunocompetent patients, the age of presentation also usually is more advanced, isolated diabetes insipidus is more common, tumor size is smaller and treatment response is more favorable (13). The case is noteworthy due to the atypical clinical presentation of a CNS lymphoma as diabetes insipidus. Confusion and diffuse neurological signs arose later on and were believed to initially be a consequence of the ionic imbalance itself rather than the mass effect. Furthermore, besides EBV negativity being highly unusual for CNS lymphomas in the HIV population, it usually portends a better prognosis, which was not observed in the reported case. Disease progression and treatment unresponsiveness were evident. Treatment-related toxicity was also significant and was the underlying cause of the patient's demise.

\section{Declaration of interest}

The authors declare that there is no conflict of interest that could be perceived as prejudicing the impartiality of the research reported.

\section{Funding}

This research did not receive any specific grant from any funding agency in the public, commercial or not-for-profit sector.

\section{Patient consent}

Written, informed consent has been obtained from a relative for the publication of this case report.
Author contribution statement

Carlos Tavares Bello: writer, patient's physician; Francisco Sousa Santos, João Sequeira Duarte and Carlos Vasconcelos reviewed the article.

\section{References}

1 Saborio P, Tipton GA \& Chan JC 2000 Diabetes insipidus. Pediatrics in Review 21 122-129; quiz 129. (doi:10.1542/pir.21-4-122)

2 Shapiro M \& Weiss JP 2012 Diabetes insipidus: a review. Journal of Diabetes and Metabolism S6 009. (doi:10.4172/2155-6156.S6-009)

3 Korfel A \& Schlegel U 2013 Diagnosis and treatment of primary CNS lymphoma. Nature Reviews Neurology 9 317-327. (doi:10.1038/ nrneurol.2013.83)

4 Sierra del Rio M, Rousseau A, Soussain C, Ricard D \& Hoang-Xuan K 2009 Primary CNS lymphoma in immunocompetent patients. Oncologist 14 526-539. (doi:10.1634/theoncologist.2008-0236)

5 Hadjizacharia P, Beale EO, Inaba K, Chan LS \& Demetriades D 2008 Acute diabetes insipidus in severe head injury: a prospective study. Journal of the American College of Surgeons 207 477-484. (doi:10.1016/j. jamcollsurg.2008.04.017)

6 Pivonello R, De Bellis A, Faggiano A, Di Salle F, Petretta M, Di Somma C, Perrino S, Altucci P, Bizzarro A, Bellastella A et al. 2003 Central diabetes insipidus and autoimmunity: relationship between the occurrence of antibodies to arginine vasopressin-secreting cells and clinical, immunological, and radiological features in a large cohort of patients with central diabetes insipidus of known and unknown etiology. Journal of Clinical Endocrinology and Metabolism $\mathbf{8 8}$ 1629-1636. (doi:10.1210/jc.2002-020791)

7 Go JL, Lee SC \& Kim PE 2006 Imaging of primary central nervous system lymphoma. Neurosurgical Focus 21 E4. (doi:10.3171/ foc.2006.21.5.5)

8 Robertson GL \& Bed T 1987 Water metabolism. In The Kidney, Chapter 11, 3rd ed, pp 385-432. Eds BM Brenner \& FC Rector. Philadelphia: Saunders.

9 Crum NF, Riffenburgh RH, Wegner S, Agan BK, Tasker SA, Spooner KM, Armstrong AW, Fraser S, Wallace MR \& Triservice AIDS Clinical Consortium 2006 Comparisons of causes of death and mortality rates among HIV-infected persons: analysis of the pre-, early, and late HAART (highly active antiretroviral therapy) eras. Journal of Acquired Immune Deficiency Syndromes 41 194-200. (doi:10.1097/01. qai.0000179459.31562.16)

10 Ahmad M 2006 Abacavir-induced reversible Fanconi syndrome with nephrogenic diabetes insipidus in a patient with acquired immunodeficiency syndrome. Journal of Postgraduate Medicine 52 296-297.

11 Engels EA, Biggar RJ, Hall HI, Cross H, Crutchfield A, Finch JL, Grigg R, Hylton T, Pawlish KS, McNeel TS et al. 2008 Cancer risk in people infected with human immunodeficiency virus in the United States. International Journal of Cancer 123 187-194. (doi:10.1002/ ijc.23487)

12 Schlegel U \& Fred H 2006 Hochberg primary CNS lymphoma. In Neuro-Oncology of CNS Tumors, Springer Berlin Heidelberg, pp 291-302. (doi:10.1007/3-540-31260-9)

13 Kaur A \& Thomas A 2015 Diffuse large B-cell lymphoma presenting with diabetes insipidus: a case report. AACE Clinical Case Reports $\mathbf{1}$ e111. (doi:10.4158/EP14295.CR)

Received in final form 3 April 2017

Accepted 7 April 2017 